\title{
Barriers and facilitators to integrating antimicrobial stewardship into clinical governance and practice: a Thai case study
}

\author{
Nantanit van Gulik ${ }^{1,6}$, Ana Hutchinson ${ }^{2,3}$, \\ Julie Considine ${ }^{2,4}$, Andrea Driscoll ${ }^{2,5}$, Kumthorn Malathu ${ }^{6}$, Mari Botti $^{2,3}$
}

\author{
1. Ramathibodi School of Nursing, Mahidol University, Bangkok, Thailand \\ 2. Centre for Quality and Patient Safety Research, Deakin University, Geelong, Victoria, Australia \\ 3. Centre for Quality and Patient Safety Research - Epworth HealthCare Partnership, Richmond, Victoria, Australia \\ 4. Centre for Quality and Patient Safety Research - Eastern Health Partnership, Box Hill, Victoria, Australia \\ 5. Austin Health, Heideberg, Victoria, Australia \\ 6. Faculty of Medicine Ramathibodi Hospital, Mahidol University, Bangkok, Thailand
}

10.3396/ijic.v16i2.013.20

\begin{abstract}
Thailand is currently facing the emergence of antimicrobial resistance. To address this issue the Royal Thai government has supported the introduction of antimicrobial stewardship (AMS) programs. Little is known about the perceptions of key-stakeholders regarding the current clinical governance of AMS and the barriers and facilitators to embedding AMS into clinical care. The aim of this study was to explore Organizational leaders' and clinical nurses' perceptions of the AMS clinical governance structure and activities at a tertiary referral centre in Thailand. Semi-structured interviews and focus group discussions were conducted with 15 Organizational leader interviews and three focus groups involving 18 nurses. Four themes emerged from the data: (1) executive seemed to endorse but not support AMS activities; (2) lack of AMS policy and resources to optimise antibiotic prescribing, tracking and reporting; (3) lack of multidisciplinary engagement in the AMS team; and (4) lack of clinician expertise and education about AMS is a major hurdle. Key issues identified included: the need to develop and embed formal AMS policies; the need for Organizational investment in personnel, information management systems, and staff education; and the need to establish a multidisciplinary approach to AMS with identifiable roles and responsibilities for each member of the team.
\end{abstract}

Keywords: antimicrobial stewardship, antimicrobial resistance, clinical governance, patient safety, Thailand

\section{Corresponding Author}

Nantanit van Gulik (Sutthiruk),

Ramathibodi School of Nursing, Faculty of Medicine Ramathibodi Hospital, Mahidol University, 270 Payathai, Ratchathewi Thailand, Bangkok 10400,

Email: nantanit.sut@mahidol.ac.th 


\section{Background}

Antimicrobial resistance (AMR) is considered by the World Health Organization (WHO) to be a major issue in healthcare. ${ }^{1}$ Antimicrobial stewardship (AMS) ensures optimal selection, dose, and duration of antimicrobial treatments to achieve the best clinical outcomes while minimising the risk of the patient experiencing side-effects and the development of AMR. ${ }^{2}$ In essence, AMS programs aim to improve patient care quality and safety through increased infection cure rates, reduced treatment failures, and correct prescribing for therapy and prophylaxis. The overall benefits of AMS are a reduction in AMR, hospital length of stay and healthcare-related costs. ${ }^{3}$

Thailand is currently facing the challenge of treating and preventing infections while reducing the emergence and spread of AMR. ${ }^{4}$ In Thailand the prevalence of extensively antibiotic resistant strains of Escherichia coli, Klebsiella pneumoniae, Acinetobacter baumannii, Pseudomonas aeruginosa, and methicillinresistant Staphylococcus aureus is increasing and is associated with increased acute care costs and patient mortality. ${ }^{4}$ To address this issue the Royal Thai government and health care services have supported a number of initiatives aimed at raising awareness of the importance of AMS in the Thai healthcare context.

In 2007, the Ministry of Public Health, Thailand launched a campaign known as Antibiotic Smart Use that promoted rational antibiotic use and supported infection control surveillance in Thai hospitals. ${ }^{5}$ Since 2013, an antibiotic awareness day has been held to promote public awareness of appropriate antibiotic use. ${ }^{6}$ In 2016, the National Strategic Plan on AMR 2017-2021 was launched by the Royal Thai Government that included the WHO Country Cooperation Strategy on $\mathrm{AMR}^{7}$, with the purpose of establishing goals for reducing morbidity and economic effects of AMR. ${ }^{6}$ Although these programs have received support from both health care administrators and a number of clinicians in Thailand, many challenges remain to embed AMS into clinical practice.

A multidisciplinary approach is an important element for initiating and sustaining AMS programs. ${ }^{3,8-10} \mathrm{An}$ Australian study found that executive level support and engagement of clinical leaders were pivotal to successful implementation of AMS programs. ${ }^{11}$ In the unique Thai healthcare context where antibiotics are widely available in the community and without prescription, ${ }^{12,13}$ little is known about organisational leaders' and clinical staff' perceptions of how clinical governance systems can be developed to support effective implementation of AMS programs. ${ }^{11}$ In addition, raising Thai clinicians' awareness of the importance of AMS is in the early stages. ${ }^{10}$ At the time this study was conducted, clinical nurses did not have a formal role in the hospital AMS governance structure and this study was undertaken to explore their current contribution to AMS and the potential to develop their role further. The aim of this study was to explore organisational leaders' and clinical nurses' perceptions of the strengths and weaknesses of the current AMS clinical governance structure and activities at a tertiary referral centre in Thailand.

\section{Methods}

Study design, sampling, and data collection.

A case study design was used to conduct this study. ${ }^{14}$ The research was conducted in a 1,000-bed university public hospital located in Bangkok, Thailand. The study hospital provides advanced medical services with approximately 5,000 outpatient visits per day and over 45,000 in-patient separations per year.

Semi-structured interviews were conducted with organisational leaders and focus group discussions with infection control nurses, senior nurses and junior nurses were used to exploring the perceived strengths and weakness of the AMS governance structure in the case study hospital. The Core Elements of a Hospital Antibiotic Stewardship Programs recommended by the Centers for Disease Control and Prevention $(C D C)^{3}$ were used to frame the semi-structured interview guide. Participants were asked about the barriers and facilitators to embedding AMS into practice specifically focusing on: the level of leadership support, access to clinical expertise, systems to track antimicrobial prescribing and antimicrobial resistance patterns, and resources to support staff education and training.

The semi-structured interviews and focus groups were conducted by one researcher ( $N v G)$ who was trained by others (MB $A D J C$ ) in qualitative 
interviewing techniques prior to conducting the interviews. After conducting the initial two interviews with organisational leaders, the interview transcripts were reviewed by the supervision team (MB $A H)$ who provided advice and feedback on interviewing techniques - specifically the use of probing and prompting techniques to elicit deeper responses from participants.

\section{Recruitment}

Purposive sampling was used to recruit participants in this study. All identified organisational leaders, infection control nurses and nurses from surgical and medical wards were invited to participate through a face-to-face invitation by the researcher. After receiving agreement to participate, suitable interview times and locations were scheduled. The interviews and focus groups were conducted in private meeting rooms at the study hospital. Focus groups were scheduled outside participants' clinical working hours, to maximise the opportunities for discussion without participants being distracted by their clinical duties. All participants provided written informed consent before starting the interview or focus group discussion.

Eighteen organisational leaders were approached and three rejected the opportunity to participate in the study as they felt that AMS was not their responsibility or a subject about which they had expertise. As 15/18 organisation leaders were included - the participation rate was $83 \%$. Purposive sampling was used to recruit the focus group participants; of the 19 nurses invited 18 participated (1 senior nurse participant missed the focus group by mistake), therefore the focus group participation rate was $95 \%$.

\section{Semi-structured interviews and \\ focus group discussions.}

There were 33 study participants, 15 individual organisational leader interviews and three focus groups involving 18 nurses were conducted between December 2016 and March 2017.

The organisational leaders interviewed were: the Hospital Director, the Director of Nursing, the Director of Pharmacy, the Chair of the Infection Prevention and Control (IPC) Committee, the IPC department nurse manager, Infection Control Nurse Specialists $(n=2)$, surgeons $(n=2)$, an infectious diseases (ID) specialist, nurse managers for the operating room and the intensive care unit (ICU), AMS clinical pharmacists $(n=2)$ and the Head of the Virology Department.

The three different groups of nurses who participated in the focus group discussions were: the Infection Control Nurse (ICN) group $(n=7)$, senior nurse $(\mathrm{SN})$ group $(n=5)$ and junior nurse $(J N)$ group $(n=6)$. The senior nurses had over 10 years of experience and the junior nurses had less than three years of experience in clinical nursing. The three focus groups were conducted separately. All focus groups were conducted by one researcher (NvG). All participants were provided with an opportunity to debrief and provide feedback.

Interviews and focus groups were recorded and transcribed for later analysis. As the participants in this study and the researcher speak Thai as their first language, the interviews and focus group discussions were conducted in Thai. Each interview and focus group was audiotaped and took approximately 30-60 minutes.

\section{Data analysis}

Thequalitativedatawereanalysed usingacombination of general inductive method and thematic analysis. ${ }^{15,16}$ Audio-recordings and field notes from the interviews were transcribed in Thai by the researcher (NvG) who conducted the interviews. The accuracy of the Thai transcripts was checked against the recordings. Next, a professional translator translated the Thai transcripts into English. The researcher (NvG) then reviewed the accuracy and consistency of the English and Thai transcripts, especially the clinical terms. Data analysis was conducted using English transcripts. To ensure the rigour of data analysis in a qualitative study, peer debriefing (with $\mathrm{MB}$ and $\mathrm{AH}$ ) occurred throughout all stages of the data analysis including the generation of the coding frame and identification of major sub-themes. Representative quotations from the transcribed text used in relation to major themes and subthemes were also confirmed. The analytical process is auditable in the raw data and coding documents.

Detailed coding was maintained throughout the analysis of the interview and focus group transcripts. 
Review of the identified themes and codes (MB, $\mathrm{JC}, \mathrm{AD}, \mathrm{AH}$ and NvG) demonstrated that thematic saturation was reached for the case study hospital.

\section{Ethical considerations}

The study was approved by the Human Research and Ethics Committees at Deakin University [Ethic Approval Number: 2015-131] and Faculty of Medicine Ramathibodi Hospital [Ethic Approval Number: MURA2015/576]. We obtained informed consent from all the participants and assured their anonymity.

\section{Results}

Participants were asked to consider the strengths and weaknesses of current clinical governance related to AMS within the hospital. The four themes that emerged from the data were: (1) executive seem to endorse but not support AMS activities; (2) lack of AMS policy and resources to optimise antibiotic prescribing, tracking and reporting; (3) lack of multidisciplinary engagement in the AMS team; and (4) lack of clinician expertise and education about AMS is a major hurdle.

\section{Theme 1. Executive seem to endorse but not support}

\section{AMS activities}

One of the barriers identified by the organisational leaders to implementing a sustainable AMS program was that the hospital executive group seem to endorse having an AMS program but did not provide additional support for program development. Subthemes related to this major theme were that (1.1) authority was delegated to clinical leaders but the executive is not actively engaged, and (1.2) IPC activities are a part of core hospital business.

\subsection{Authority was delegated to clinical leaders but the} executive is not actively engaged. There were mixed perceptions of executive support for AMS projects. In general, all of the key organisational leaders, perceived that the hospital administrators had endorsed the introduction of the AMS program.

I think the hospital director fully supports [AMS]. At the moment, we are focusing on Antibiotic Smart Use in the Rational Drug Use program. (Clinical Leader 6)

I think that the senior hospital administrators are very supportive. We know that ID specialists have been given authority to control antibiotic use in the hospital. If they [ID specialists] find antibiotics are prescribed inappropriately they can take action. (Clinical Leader 4)

Participants in the senior nurses and ICN focus groups also reported that the hospital executive team was aware of the AMR problem and had taken action to limit carbapenem (a class of broad spectrum antibiotic used to treat more resistant organisms) prescribing across the organisation. The chairperson of the IPC committee was identified as the person responsible for leading new AMS initiatives at the study hospital.

I think the hospital executive is aware of the AMR problem. You can firstly see from the program controlling use of antibiotics such as carbapenems, in which general doctors are limited to prescribing [this antibiotic] for only three days. After that, they have to consult an ID specialist. (SN3)

I think the hospital director fully supports [AMS programs] but the chairman [of the IPC and the Antibiotic and Vaccine committees] has to put projects forward. (ICN3)

Despite this, there was a perception that senior hospital executives were not fully supportive of the AMS program as they did not provide additional financial resources to support program development.

It [AMS leadership support] seems good but I'm not sure because there is no additional compensation and staff for the AMS team. (Clinical Leader 1)

Personally, I don't think the senior executives are fully supportive of AMS programs. I found that only Dr. [name] is active in the AMS program ..... Also, the hospital executive committee provides just intermittent support. (Clinical Leader 7)

The existence of a strong clinical champion who was a recognised, expert ID specialist and chair of the IPC committee was identified as a strength of the current AMS program.

Personally, I think because the IPC chairman is a doctor and has authority to approach the hospital 
executive committee; he is able to convince them that they should be concerned about this [AMR] problem along with how to implement IPC and AMS policies within the hospital. (Clinical Leader 11)

\subsection{IPC activities are a part of core hospital business.} In contrast to the intermittent support provided for AMS programs, the organisational leaders recognised that there was strong executive-level support for IPC activities and that these activities were perceived as part of core hospital business.

Of course, we [the hospital executive] are very supportive. The IPC committee has the role in proposing the project, and we will find the best solution. (Clinical Leader 2 )

I think the hospital executive committee supports us very well especially in relation to IPC activities, for example, the hand hygiene campaign. I have never heard that they rejected even one project. (Clinical Leader 11)

Once we had an outbreak of VRE [vancomycinresistant enterococcus] in the hospital, which meant that everyone in the hospital was on full alert. From that experience, we also found that the senior executive launched quickly into action implementing a policy of strict patient isolation and infection control precautions along with controlling vancomycin prescribing. (Clinical Leader 13)

\section{Theme 2 Lack of AMS policy and resources to optimise} antibiotic prescribing, tracking and reporting

The second major theme identified that there was a lack of formal AMS policies and resources to optimise, track and enforce good antimicrobial prescribing practice. Related subthemes were:(2.1) no formal AMS policy and local antibiotic guidelines, (2.2) inadequate information technology (IT) infrastructure to support optimal antibiotic prescribing, and (2.3) gaps in the current tracking and reporting of antimicrobial use.

2.1 No formal AMS policy and local antibiotic guidelines. Organisational leaders and clinical nurses perceived that an important barrier to effective implementation of AMS programs was the lack of formal AMS policies and antibiotic guidelines that were specific to the
Thai context. This was considered to be an important deficit in the available resources and decreased prescribers' confidence in the relevance of the guidelines to their clinical practice. In contrast, it was identified that the organisational guidelines for antiviral use were evidence-based and locally relevant and that this supported good practice in anti-viral use at the study site.

They [the senior executives] have not been consistent in establishing a formal AMS policy. This is a very important barrier [to effective program implementation]. (Clinical Leader 7)

We have national anti-viral drug use guidelines and a clear workflow plan that is regularly updated,.... it is a very useful reference. However, there is no guideline for antibiotics which doctors from all departments are able to [consult when] prescribing, ....when guidelines are absent, it is hard to control antibiotic use. (Clinical Leader 15)

Participants in the junior nurses focus group reported a lack of clarity regarding how the principles of AMS should be incorporated into clinical bedside care.

I think they give priority to the issue of AMS, but the policy that is communicated to frontline staff is still not clear, it only raises awareness. (JN4)

2.2 Inadequate IT infrastructure to support optimal antibiotic prescribing. The participants discussed the problem of limited IT infrastructure to support AMS activities. It was thought that the current IT system did not have the capacity to link patients' prescribed medications to their microbiology results and provide the bedside clinician with real-time decision-support.

We heard that the AMS team is trying to develop antibiotic prescribing guidelines ...Once we had some training about antibiotic use and we got some antibiotic use guidelines as a paper copy which was easy to lose. It would be better if we have a smart application or decision-support software on the hospital intranet that we can access everywhere, whenever we prescribe antibiotics. (Clinical Leader 5) 
The missing program right now is the program that links the data between the antibiotic prescribed and the microbiology laboratory reports of [antimicrobial] susceptibilities. (Clinical Leader 7)

Junior nurses highlighted that they needed information systems that would highlight whether the antibiotics prescribed were appropriate once the pathology results became available.

If we had a system that identified particular bacteria and could indicate which antibiotics we should give to the patient [that match that organism's bacterial sensitivities] that would help us. (JN4)

\subsection{Gaps in the current tracking and reporting of antimicrobial use.}

Both the organisational leaders and clinical nurses interviewed identified gaps in the current tracking and reporting of AMS activities.

We do not have a systematic approach to reporting antibiotic use [to the clinical staff]. (Clinical Leader 12)

Dr. [name] is monitoring antibiotic use in the hospital but we do not systematically report either the volume of antibiotic use or the [incidence of] multiple drugresistant organisms. Personally, I would like the data to be reported to frontline staff such as nurses. (Clinical Leader 14)

These days we do not receive a report about antibiotic use in the hospital that is specific enough. I believed that every doctor would be interested in the overall use of antibiotics. Last time we [surgeons] received the surgical site infections report, I noticed that they all looked so excited to see their operation outcomes reported. (Clinical Leader 5)

Clinical nurses reported that the lack of systems to support monitoring and tracking of antibiotic prescribing was hampering efforts to implement a sustainable AMS program.

We know that there is a serious problem regarding antimicrobial resistance in the hospital,... for example, when there was an outbreak of VRE [vancomycin resistant enterococcus], we were on high alert... But we do not have an overview of the infections in the hospital overall. (JN6)

At the moment, we have not yet received a report about the number of antibiotics we used and how much it cost. It would be good if we had those reports made available. It may make us more aware of using antibiotics wisely and we don't want to waste money. (SN4)

Additionally, a doctor participant suggested that an audit and feedback system was needed to improve prescriber behavior.

I would like to have the prospective audit and feedback system that included annual reporting. Say, each year every department receives feedback, an antibiotic prescribing report. ... I believe everyone wants his or her patients to experience AMR infections as little as possible. If we don't start taking action and no feedback is provided, we will never know when our prescribing practice is poor, and everyone has only excuses [for their prescribing choices]. (Clinical Leader 5 )

\section{Theme 3 Lack of multidisciplinary engagement in AMS team}

The third major theme to emerge was the lack of multidisciplinary engagement in the AMS team. The identified sub-themes were: (3.1) not an ideal AMS team and training, (3.2) lack of physician engagement in AMS and, (3.3) divergent opinions about the participation of nurses in AMS.

3.1 Not an ideal AMS team and training. The lack of multidisciplinary engagement in AMS was identified as a gap in the current leadership commitment and this was perceived as an obstacle to the effective promotion of the AMS program

They [the hospital executive] do not allocate pharmacists or nurses to be part of the AMS team. It is not an ideal AMS team right now. It's not an AMS system like other countries that have a full-time job for members of the AMS team. (Clinical Leader 1)

It seems like each profession has their own training, but there is no multidisciplinary training conference... 
in which, an ID specialist educates staff about antimicrobial resistance, a microbiologist presents on the microbiology of AMR, and pharmacists talk about dose adjustment (Clinical Leader 7)

\subsection{Lack of physician engagement in AMS.} Organisational leaders and clinical nurses identified a lack of physician engagement was one of the barriers to multidisciplinary involvement in AMS.

Although we realised that the senior executives prioritise this issue [AMS] more than before, there is still a group of doctors that use antibiotics improperly even though several antibiotics have restricted prescribing. (Clinical Leader 10)

I think that the senior executives have not been strong enough to control antibiotic prescribing... Some doctors still use high-level broad-spectrum antibiotics for surgical prophylaxis, even though the hospital leaders have discussed this with them... They do not care and keep on prescribing that antibiotic. (Clinical Leader 12)

Both organisational leaders and junior nurses identified that challenging prescribers' decisionmaking was difficult, as doctors did not like their authority being questioned and would not be willing to receive feedback about their prescribing practice from other professional groups.

Most importantly, in our country, doctors have too much power, so there would be no acceptance of other professional groups [becoming involved in AMS] if it [the extension of their role] would interfere with doctors' [professional] boundaries. (Clinical Leader 1)

I think it's about the power to make a decision, like, when we [nurses] notice that a doctor has prescribed high dose antibiotics, we [the clinical nurses] and the pharmacist tried to remind the doctor [that this is not ideal practice], but he/she insisted on the same order. Some doctors have huge egos. They feel that they lose authority when we oppose them. (JN4)

3.3 Divergent opinions about the participation of nurses in AMS. There were differences of opinions about whether nurses should be involved in AMS governance committees, specifically the Antibiotic and Vaccine
Committee that oversees antimicrobial availability and use across the hospital. A number of participants explained that the purpose of these committees was not directly relevant to nursing practice.

Personally, I thought that it is okay if nurses are not involved in the [Antibiotic advisory] committee because they consider what antibiotics should be available in the hospital. The committee members consider in detail antimicrobial pharmacodynamics, which might not relate to nursing knowledge. If nurses would like to get involved in this committee those nurses should have an in-depth knowledge of antibiotic pharmacology. (Clinical Leader 4)

Currently, nurses have a role in infection and prevention control committee where they monitor prevention and control of antimicrobial resistance. (Clinical Leader 1)

In contrast, others thought that as nurses were responsible for patient safety they should be represented and would make a valuable contribution. The Pharmaceutical Consideration sub-committees do not include nurses, because it is all about considering the list of medicines available in the hospital. Actually, nurses were in those groups, but they said there is nothing related to their role.... However, I agree that nurses should be included in the Antibiotics and Vaccines committee because there are other activities discussed that are quite related to nursing care. (Clinical Leader 6)

If the Antibiotics and Vaccines committee how medications are administered such as how to drip [administer] each antibiotic. Nurses should be involved because all of the multidisciplinary team should be. (Clinical Leader 4)

Both nurse leaders and junior nurses agreed that nurses should be represented on key governance committee that support AMS as nurses would provide important clinical insights, and would highlight the safety implications of policy changes at a ward level.

In my opinion, if this team [the Antibiotics and Vaccines committee members] have pharmacists, they should have a nurse as well....My question is whether they [pharmacists] know more about the patients or not? 
Do they check the lab results? I do not think so. Thus, I think this team should have physicians, nurses, and pharmacists working together. (Clinical Leader 10)

I think we should have nurses on this committee [the Antibiotics and Vaccines committee] because nurses are involved with administering antibiotic therapy 24 hours/day. We know the patients' condition,... we know what medications they are allergic to. (JN6)

I think [the committee] should have nurses from different levels on this board because sometimes the executives do not know the problems or things we really encounter [on the ward], even ICNs do not work with the patients all the time. (JN4)

Infection control nurses were recognised as crucial members of the AMS committee as they have expertise in infection surveillance and experience monitoring antibiotic use.

I would like to have at least one infection control nurse involved in the Antibiotics and Vaccines team because infection control nurses do AMR surveillance in the hospital. That would give us continuity and clarity to support the AMS work. (Clinical Leader 11)

Nurses who participate in this board [Antibiotics and Vaccines committee] should be senior nurses such as ward charge nurses. I think an ICN should also participate in order to update [staff] on the situation ....and to see the whole picture of antibiotic use across the hospital. (ICN1)

\section{Theme 4 Lack of clinician expertise and education about AMS is a major hurdle}

The fourth major theme that emerged was that the majority of clinicians (fromacross arange of disciplines) lacked expertise in AMS and that this represented a major hurdle to embedding the principles of AMS into routine clinical care. The associated sub-themes were that (4.1) knowledge is acquired through experience not education and, (4.2) need for an organisationwide, multidisciplinary approach to AMS education.

4.1 Knowledge acquired through experience, not education. Knowledge gaps relating to appropriate antibiotic use were identified across all members of the multidisciplinary team. Only relatively small numbers of experienced clinicians were recognised as having sufficient expertise in AMS to guide their practice.

[As they have more knowledge...] ID specialists and paediatrics are more concerned about antibiotic use. Surgical doctors and gynaecologists have less knowledge about this issue. (Clinical Leader 11)

I have not much knowledge in antibiotic use, particularly the mechanisms of AMR, how to use antibiotics appropriately to reduce AMR, also the best route of antibiotic administration to use. (Clinical Leader 5)

I have noticed that on weekends broad-spectrum antibiotics are prescribed a lot. Then on Monday the ID fellows and staff come and change that. (Clinical Leader 14)

Nurse leaders reported that although nurses had expertise in IPC, they lacked the knowledge to evaluate whether antibiotic prescribing choices were appropriate.

Nurses have good knowledge about how to isolate patients who have infectious diseases. Probably they [nurses] have more knowledge than doctors in isolation precautions. But nurses do not have much knowledge about antibiotic use. (Clinical Leader 11)

Nurses and pharmacists also identified that clinicians' expertise regarding the principles of AMS and appropriate antibiotic use was dependent on clinical speciality and work experience rather than formal education.

Actually, from my experience, if we regularly use particular antibiotics for specific patient types, we eventually remember it [the indication for antibiotics], but [our knowledge] is not in-depth. (SN2)

About antibiotic use, nurses who take care of patients who have AMR infections would be more knowledgeable. For example, when there was CRE [carbapenem resistant Enterobacteriaceae] outbreak in the hospital, nurses would know that [a contributing 
factor] was that patients had used carbapenem [broad spectrum antibiotic used to treat resistant infections] inappropriately. Or when there was a VRE outbreak, nurses knew that this was caused by vancomycin use...

\section{(Clinical Leader 14)}

Not all pharmacists know all about antibiotic use because they get less involved with AMR pathogens. Only clinical pharmacists have expertise in antibiotics. But they would know more about antibiotics than nurses. (Clinical Leader 7)

\subsection{Need for an organisation-wide, multidisciplinary} approach to AMS education. Participants were supportive of the hospital providing a multidisciplinary education program to increase staff awareness, knowledge and skills in relation to management of patients with AMR and how to apply AMS principles in their clinical practice.

It seems like each profession has their training, but there is no multidisciplinary training or a multidisciplinary conference. We have not yet had a conference in which an ID specialist educates about antimicrobial resistance, a microbiologist presents microbiology, and pharmacists talk about dose adjustment. (Clinical Leader 7)

We should have the conference like the IPC conference which has doctors, nurses, and pharmacists as speakers annually. (Clinical Leader 5)

We should have the campaign to empower personnel to see the importance of it [antibiotic use and resistance]. (ICN1)

\section{Discussion}

The study participants perceived both strengths and weaknesses in the current clinical governance structures at the study hospital. Strong executive level support for IPC activities and having a central champion for AMS activities were identified as advantageous and helped promote the importance of AMS to both clinicians and the hospital executive. Despite this perceived strength, a number of important barriers to the implementation of a sustainable, organisation-wide AMS program were identified. Participants perceived that the health service executive acknowledged the importance of tackling the issue of AMR and therefore endorsed the roll-out of a number of AMS projects at the study hospital. There was not however organisational investment or the provision of sufficient resources to ensure that these projects were sustainable.

To initiate AMS programs, international guidelines suggest that a clinical champion such as an ID physician and/or clinical pharmacist provide leadership as a minimum requirement for an effective AMS program. ${ }^{3,8,11,17-20}$ As the local AMS champion is a recognised expert in the field, it was easier to convince clinicians to support AMS activities. ${ }^{21-23}$ As this champion was also the IPC committee chair this strengthened the integration of IPC and AMS activities at the study site. There were however some concerns expressed that this had the potential to underplay the importance and contribution of nurses.

The CDC recommendations for the core elements of AMSprogramssuggest that the hospital administrators should provide all clinicians with ease of access to current prescribing guidelines ${ }^{3,21,24,25}$ Previous studies suggest that implementing clinical guidelines that take into account local microbiology and antimicrobial susceptibility patterns is an essential element in an effective AMS strategy. ${ }^{21,25}$ At the study hospital inadequate IT resources to support decision-making and monitoring of antimicrobial use was reported as a gap in the local AMS program. ${ }^{9,21,26}$ A recurring theme was that accessing expertise and decision-support tools was difficult in a busy clinical environment and participants indicated that they needed additional access to locally relevant decision-support software at the bedside.

International AMS guidelines such as those from the USA, Australia, Scotland, and the UK recommend that a hospital-wide multidisciplinary approach is best practice in AMS programs. ${ }^{3,8,24,27,28}$ The study participants identified a range of important barriers that need to be addressed before a sustainable, organisation-wide, comprehensive AMS program is established at the study hospital. ${ }^{27,29}$ Key issues that were identified included: the need to develop formal AMS policies and to embed these into the clinical governance structure; the need for organisational 
investment in personnel, information management systems, and staff education; and the need to establish a multidisciplinary approach to AMS with identifiable roles and responsibilities for each member of the team.

These recommendations complement the OneHealth approach to tackling antimicrobial resistance in Thailand that was adopted by the Royal Thai government in 2017, in which a multidisciplinary and cross-sectoral approach to tacking AMR was endorsed. ${ }^{30}$ At a national level the introduction of surveillance systems to monitor antimicrobial consumption and the incidence of AMR in both human and animal population in Thailand is recommended. ${ }^{30}$ Although this policy document identified the importance of raising awareness of AMR across all sectors of the Thai community, it was recognised that for real, sustained change to occur, regulatory authorities needed to have greater capacity to enforce AMS policy implementation.

\section{Conclusions}

The major findings of this study are that although there is an executive level endorsement of AMS projects and there is a recognised clinical champion who provides strong leadership for AMS activities across the organisation, that the AMS program had not yet matured to the stage where it is fully integrated into the clinical governance structure of the organisation. The findings also highlighted that the AMS programs had not reached clinicians from different disciplines to the extent necessary for their engagement and consistent implementation in clinical practice.

\section{Acknowledgements}

The authors wish to thank all the participants who contributed to this study.

Potential conflicts of interest. All authors have no conflicts of interest relevant to this study.

\section{References}

1. WHO. Antimicrobial resistance. 2017. http://www.who.int/ mediacentre/factsheets/fs194/en/. Accessed 1 October 2017, 2017.

2. Gerding DN. The search for good antimicrobial stewardship. The Joint Commission journal on quality improvement 2001; 27: 403-404. https://doi.org/10.1016/S1070-3241(01)27034-5
3. CDC, ed Core Elements of Hospital Antibiotic Stewardship Programs Atlanta, GA: US Department of Health and Human Services; 2014.

4. Pumart P, Phodha T, Thamlikitkul V, Riewpaiboon A, Prakongsai P, Limwattananon S. Health and economic impacts of antimicrobial resistance in Thailand. Journal Health Services Research Policy 2012; 9: 352-360.

5. MOPH. Antibiotic Resistance: Crisis of Thai People's Health. Paper presented at: the National Seminar on "Antibiotic Resistance: Crisis of Thai People's Health; 28 - 29 May 2012, 2012; Bangkok, Thailand.

6. Sumpradit N, Wongkongkathep S, Poonpolsup S, et al. New chapter in tackling antimicrobial resistance in Thailand. BMJ 2017; 358. doi: https://doi.org/10.1136/bmj.j2423

7. WHO. WHA 68.7 agenda item: Global action plan on antimicrobial resistance. 2015. http://apps.who.int/gb/ ebwha/pdf_files/WHA68/A68_R7-en.pdf. Accessed 10 October, 2019.

8. Duguid M, Cruickshank M. Antimicrobial stewardship in Australian hospitals. Sydney: Australian Commission on Safety and Quality in Health Care; 2011.

9. Dellit TH, Owens RC, McGowan JE Jr, et al. Infectious Diseases Society of America and the Society for Healthcare Epidemiology of America guidelines for developing an institutional program to enhance antimicrobial stewardship. Clinical Infectious Diseases 2007: 159. https://doi. org/10.1097/IPC.0b013e318068b1c0

10. Sutthiruk N, Considine J, Hutchinson A, Driscoll A, Malathum $\mathrm{K}$, Botti M. Thai clinicians' attitudes toward antimicrobial stewardship programs. American Journal of Infection Control 2018; 46: 425-430. https://doi.org/10.1016/j. ajic.2017.09.022

11. Loh JAM, Darby JD, Daffy JR, et al. Implementation of an antimicrobial stewardship program in an Australian metropolitan private hospital: lessons learned. Healthcare Infection 2015; 20: 134-140. https://doi.org/10.1071/ HI15015

12. Sommanustweechai A, Yeung S, Chanvatik $S$, et al. Antibiotic distribution channels in Thailand: Results of key-informant interviews, reviews of drug regulations and database searches. Bulletin of the World Health Organisation 2018; 96: 101-109. https://doi.org/10.2471/BLT.17.199679

13. Sutthiruk N, Considine J, Hutchinson A, Driscoll A, Malathum $\mathrm{K}$, Botti M. A survey of reported behaviours, attitudes and knowledge related to antibiotic use of hospitalised patients in Thailand. Infection, Disease \& Health 2018; 23: 203-210. https://doi.org/10.1016/j.idh.2018.05.002

14. Crowe S, Cresswell K, Robertson A, Huby G, Avery A, Sheikh A. The case study approach. BMC medical research methodology 2011; 11: 100. https://doi.org/10.1186/1471-2288-11-100

15. Saks M, Allsop J. Researching health: qualitative, quantitative and mixed methods. London: SAGE, 2012.

16. Braun V, Clarke V. Using thematic analysis in psychology. Qualitative Research in Psychology 2006; 3: 77-101. https:// doi.org/10.1191/1478088706qp063oa

17. Doron S, Davidson LE. Antimicrobial Stewardship. Mayo Clinic Proceedings 2011; 86: 1113-1123. https://doi.org/10.4065/ mcp.2011.0358 
18. Cotta MO, Robertson MS, Marshall C, Thursky KA, Liew $\mathrm{D}$, Buising KL. Implementing antimicrobial stewardship in the Australian private hospital system: a qualitative study. Australian Health Review 2015; 39: 315-322. https://doi. org/10.1071/AH14111

19. Septimus EJ, Owens RC, Jr. Need and potential of antimicrobial stewardship in community hospitals. Clinical Infectious Diseases 2011; 53: S8-S14. https://doi.org/10.1093/cid/cir363

20. Yam P, Fales D, Jemison J, Gillum M, Bernstein $M$. Implementation of an antimicrobial stewardship program in a rural hospital. American Journal of Health-System Pharmacy 2012; 69: 1142-1148. https://doi.org/10.2146/ajhp110512

21. Pakyz AL, Moczygemba LR, VanderWielen LM, Edmond MB, Stevens MP, Kuzel AJ. Facilitators and barriers to implementing antimicrobial stewardship strategies: Results from a qualitative study. American Journal Of Infection Control 2014; 42: S257-S263. https://doi.org/10.1016/j. ajic.2014.04.023

22. Jeffs L, Thampi N, Maione M, Steinberg M, Morris AM, Bell CM. A Qualitative analysis of implementation of antimicrobial stewardship at 3 academic hospitals: understanding the key influences on success. The Canadian journal of hospital pharmacy 2015; 68: 395-400. https://doi.org/10.4212/cjhp. v68i5.1486

23. Pollack LA, Srinivasan A. Core elements of hospital antibiotic stewardship programs from the Centers for Disease Control and Prevention. Clinical infectious diseases: an official publication of the Infectious Diseases Society of America 2014; 59 Suppl 3: S97-100. https://doi.org/10.1093/cid/ciu542

24. Nathwani D. Antimicrobial prescribing policy and practice in Scotland: recommendations for good antimicrobial practice in acute hospitals. Journal of Antimicrobial Chemotherapy (JAC) 2006; 57: 1189-1196. https://doi.org/10.1093/jac/dkl137
25. James RS, Mclntosh KA, Luu SB, et al. Antimicrobial stewardship in Victorian hospitals: a statewide survey to identify current gaps. Med J Aust 2013; 199: 692-695. https:// doi.org/10.5694/mja13.10422

26. Buising KL, Thursky KA, Black JF, et al. Improving antibiotic prescribing for adults with community acquired pneumonia: Does a computerised decision support system achieve more than academic detailing alone?--A time series analysis. BMC Med Inform Decis Mak 2008; 8: 35. https://doi. org/10.1186/1472-6947-8-35

27. Lin Y-S, Lin IF, Yen Y-F, et al. Impact of an antimicrobial stewardship program with multidisciplinary cooperation in a community public teaching hospital in Taiwan. AJIC: American Journal of Infection Control 2013: 1069. https://doi. org/10.1016/j.ajic.2013.04.004

28. SACAR. Antimicrobial Framework. Journal of Antimicrobial Chemotherapy (JAC) 2007; 60: i87-i87. https://doi. org/10.1093/jac/dkm185

29. Cotta MO, Robertson MS, Tacey M, et al. Attitudes towards antimicrobial stewardship: results fromalarge private hospital in Australia. Healthcare Infection 2014; 19: 89-94. https://doi. org/10.1071/HI14008

30. Sommanustweechai A, Tangcharoensathien V, Malathum $\mathrm{K}$, et al. Implementing national strategies on antimicrobial resistance in Thailand: potential challenges and solutions. Public Health 2018; 157: 142-146. https://doi.org/10.1016/j. puhe.2018.01.005 